Article

\title{
Efficient Synthesis of New Fluorinated $\beta$-Amino Acid Enantiomers through Lipase-Catalyzed Hydrolysis
}

\author{
Sayeh Shahmohammadi ${ }^{1}$, Ferenc Fülöp ${ }^{1,2}$ [D and Enikő Forró ${ }^{1,2, *}$ \\ 1 Institute of Pharmaceutical Chemistry, University of Szeged, H-6720 Szeged, Hungary; \\ sayeh.s@pharm.u-szeged.hu (S.S.); fulop@pharm.u-szeged.hu (F.F.) \\ 2 Stereochemistry Research Group of the Hungarian Academy of Sciences, University of Szeged, \\ H-6720 Szeged, Hungary \\ * Correspondence: forro.eniko@pharm.u-szeged.hu; Tel.: +36-62-544964; Fax: +36-62-545705
}

Received: 27 October 2020; Accepted: 14 December 2020; Published: 17 December 2020

\begin{abstract}
An efficient and novel enzymatic method has been developed for the synthesis of $\beta$-fluorophenyl-substituted $\beta$-amino acid enantiomers through lipase PSIM (Burkholderia cepasia) catalyzed hydrolysis of racemic $\beta$-amino carboxylic ester hydrochloride salts $3 \mathbf{a}-\mathbf{e}$ in $i \operatorname{Pr}_{2} \mathrm{O}$ at $45{ }^{\circ} \mathrm{C}$ in the presence of $\mathrm{Et}_{3} \mathrm{~N}$ and $\mathrm{H}_{2} \mathrm{O}$. Adequate analytical methods were developed for the enantio-separation of racemic $\beta$-amino carboxylic ester hydrochlorides $3 \mathbf{a}-\mathbf{e}$ and $\beta$-amino acids $2 \mathbf{a}-\mathbf{e}$. Preparative-scale resolutions furnished unreacted amino esters $(R)-\mathbf{4 a}-\mathbf{e}$ and product amino acids (S)-5a-e with excellent $e e$ values ( $\geq 99 \%)$ and good chemical yields ( $>48 \%)$.
\end{abstract}

Keywords: kinetic resolution; lipase-catalyzed hydrolysis; enantioselective synthesis; fluorinated $\beta$-amino acid

\section{Introduction}

In recent years, enantiomerically pure $\beta$-aryl-substituted $\beta$-amino acids have been intensively investigated due to their pharmacological significance, unique and remarkable biological properties [1], their utility in synthetic chemistry [2], and drug research [3]. Therefore, this class of compounds has been documented as a crucial scaffold in the design and synthesis of conceivable pharmaceutical drugs. For instance, 3-amino-3-phenylpropionic acid, which is a key pharmaceutical building block, is present in anticancer agents, such as Taxol [4]. It can also find application as a fundamental component in the synthesis of novel antibiotics [5] and analgesic endomorphine-1 analogue tetrapeptides [6].

On the other hand, tremendous achievements in the development of fluorinated amino acid drugs verified the high importance of this type of compounds in pharmaceutical chemistry. It is known that the occurrence of fluorine in biologically active natural compounds is extremely low. In turn, the number of fluorine-containing drugs on the market is rising continuously. The reasons are the unique characteristics of the fluorine atom in terms of its high electronegativity and the polarity of a carbon-fluorine bond $[7,8]$. Thus, incorporation of fluorine into $\beta$-amino acids has gained increasing attention in recent decades. For example, Januvia (sitagliptin phosphate) acts as an antidiabetic agent via inhibition of dipeptidyl peptidase IV [9], whereas ( \pm )-Eflornithine was used for the treatment of trypanosomiasis [10] and against facial hirsutism in women [11]. 
There are different approaches for the synthesis of optically active $\beta$-aryl- $\beta$-amino acids [12-14]. The utilization of enzymes in these reactions gained special attention, which is due to their ability to conduct the reactions enantio-selectively. For example, lipases are stable. They work under mild conditions and many of them are commercially available. They can be applied on an industrial scale $[15,16]$. Lipase-catalyzed methods for the resolution of both cyclic [17] and acyclic [18] $\beta$-amino carboxylic esters through hydrolysis are known in the literature. Various enzymatic procedures have been developed by our research group for the preparation of biologically active $\beta$-aryl-substituted, $\beta$-heteroaryl-substituted, and $\beta$-arylalkyl-substituted $\beta$-amino acid enantiomers through enantioselective $(E>200)$ hydrolysis of the corresponding $\beta$-amino carboxylic esters both in $\mathrm{H}_{2} \mathrm{O}$ or in an organic solvent catalyzed by lipase (Pseudomonas cepacia) PS[19-21]. Catalyzed kinetic and dynamic kinetic resolution of $\beta$-amino carboxylic esters or their hydrochloride salts with tetra-hydro-isoquinoline and tetra-hydro- $\beta$-carboline skeleton through hydrolysis have been performed. Catalysts used include Candida antarctica lipase B (CAL-B) (in aqueous $\mathrm{NH}_{4} \mathrm{OAc}$ buffer at pH 8.5 and or in $i \mathrm{Pr}_{2} \mathrm{O}$ in the presence of 1 equiv of $\mathrm{H}_{2} \mathrm{O}$ ), Alcalase (in borate buffer at $\mathrm{pH}$ 8), and lipase PS (in $i \operatorname{Pr}_{2} \mathrm{O}$ with 4 equiv of added $\mathrm{H}_{2} \mathrm{O}$ ) [22-25].

A number of $\mathrm{N}$-benzylated $\beta^{2}-, \beta^{3}-$, and $\beta^{2,3}$-amino acids were prepared through CAL-B-catalyzed hydrolysis of the corresponding racemic amino carboxylic esters with $\mathrm{H}_{2} \mathrm{O}$ in n-hexane or 2-methyl-2-butanol, under stirring [26] or utilizing high-speed ball-milling conditions [27]. Covalently immobilized lipase AK (Pseudomonas fluorescens) and lipase PS were used as efficient stereoselective catalysts for the kinetic resolution of exotic and variously substituted rac-(5-phenylfuran-2-yl)- $\beta$-alanine ethyl ester hydrochlorides through hydrolysis $(E>146)$ in $\mathrm{NH}_{4} \mathrm{OAc}$ buffer $(20 \mathrm{mM}, \mathrm{pH} 5.8)$ at $30^{\circ} \mathrm{C}$ [28]. Gotor et al. reported the kinetic resolution $(E>200)$ of a large number of 3-amino-3-phenylpropanoate esters through lipase PS-catalyzed hydrolysis with $\mathrm{H}_{2} \mathrm{O}$ in 1,4-dioxane at $45^{\circ} \mathrm{C}$ [18]. The method was successfully used for the synthesis of (S)-3-amino-3-phenylpropionic acid, which is a key precursor for the preparation of (S)-dapoxetine, and a potent selective serotonin reuptake inhibitor (SSRI) used for the treatment of depression, bulimia, or anxiety [29]. In addition, very recently, Zhang et al. summarized a review of the most facile catalytic enantioselective strategies to construct the fluorinated $\alpha$-amino and $\beta$-amino acids [30].

Herein, in view of the importance of fluorinated $\beta$-amino acids, our aim was to synthesize $( \pm)$ - $\beta$-amino carboxylic ester hydrochloride salts $\mathbf{3 a}-\mathbf{e}$ (Scheme 1$)$, then to devise a suitable enzymatic protocol for the synthesis of new fluorinated $\beta$-amino acids via enantioselective hydrolysis of $\mathbf{3 a}-\mathbf{e}$ (Scheme 2) and provide an adequate characterization of the enantiomeric products.

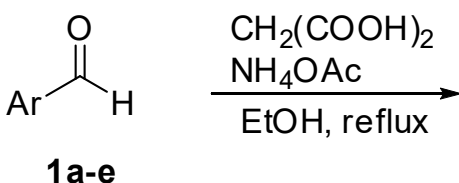

$1 \mathrm{a}-\mathrm{e}$

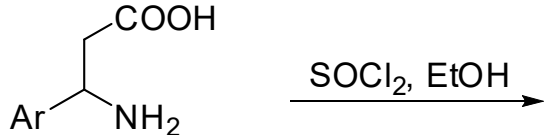

$( \pm)-2 \mathrm{a}-\mathrm{e}$<smiles>CCOC(=O)CC(N)Br</smiles>

$( \pm)-3 a-e$

Ar:<smiles>Cc1ccc(F)c(F)c1</smiles><smiles>Cc1cc(F)cc(F)c1</smiles><smiles>Cc1ccc(F)cc1</smiles><smiles>Cc1ccc(C(F)(F)F)cc1F</smiles><smiles>Cc1ccc(C)c(F)c1</smiles>

Scheme 1. Synthesis of ( \pm )-3a-e. 
<smiles></smiles>

( \pm -3a-e

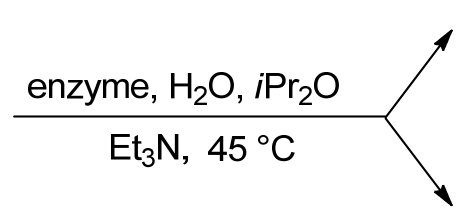<smiles>[R]c1cc([C@H](N)CC(=O)OCC)c([R])c([R])c1[R]</smiles>

$(R)-4 a-e$<smiles>[R]c1cc(C(N)CC(=O)O)c([R])c([R])c1[R]</smiles>

(S)-5a-e

3a: $R^{2}=R^{3}=F, R^{1}=R^{4}=H$

3b: $R^{2}=R^{4}=F, R^{1}=R^{3}=H$

3c: $R^{1}=R^{2}=R^{4}=H, R^{3}=F$

3d: $R^{1}=F, R^{3}=C F_{3}, R^{2}=R^{4}=H$

3e: $R^{1}=F, R^{3}=\mathrm{CH}_{3}, R^{2}=R^{4}=H$

Scheme 2. Enzymatic kinetic resolution of ( \pm )-3a-e through a hydrolytic procedure.

\section{Results}

\subsection{Synthesis of Ethyl 3-Amino-3-Arylpropanoate Hydrochloride Salts ( \pm )-3a-e}

Racemic $\beta$-amino acids ( \pm -2a-e were synthesized by modified Rodionov synthesis through the reaction of the corresponding aldehydes with malonic acid in the presence of $\mathrm{NH}_{4} \mathrm{OAc}$ in $\mathrm{EtOH}$ at a reflux temperature (Scheme 1) [31,32]. Subsequently, the $\beta$-amino carboxylic ester hydrochloride salts $( \pm)-3 a-e$ were prepared with yields ranging from $76 \%$ to $98 \%$ by esterification of the corresponding $\beta$-amino acids in the presence of $\mathrm{SOCl}_{2}$ in $\mathrm{EtOH}$.

\subsection{Enzyme-Catalyzed Hydrolysis of ( \pm -3a-e}

\subsubsection{Preliminary Experiments}

On the basis of the results achieved on the enzyme-mediated enantioselective hydrolysis of $\beta$-amino carboxylic esters [17,18], the hydrolysis of model compound ( \pm )-3a (Scheme 2) was conducted with 5 equiv of $\mathrm{Et}_{3} \mathrm{~N}$ and 0.5 equiv of $\mathrm{H}_{2} \mathrm{O}$ in the presence of $30 \mathrm{mg} \mathrm{mL}^{-1}$ enzyme in $i \operatorname{Pr}_{2} \mathrm{O}$ at $45^{\circ} \mathrm{C}$ (Table 2, entry 1). In the frame of enzyme screening, lipase AY (Candida rugosa), lipase AK, PPL (Porcine pancreatic lipase), and CAL-B (Table 2, entries 2-5) showed activity in enzymatic hydrolysis. However, with the exception of lipase AK affording an $e e_{\mathrm{p}}$ value of $75 \%$ and a moderate $E(8)$ (19\% conversion in $10 \mathrm{~min}$, entry 3), low reactivities and low enantio-selectivities were achieved (entries 2, 4, and 5). It is noteworthy that PPL catalyzed the reaction with opposite enantio-selectivity. Lipase PSIM, in contrast, provided an E value of 108 (entry 1) and, consequently, it was selected for further studies.

Next, we analyzed the effect of solvent on enantio-selectivity and reaction rate. Very different $E$ and reaction rate data were observed in the green solvents tested (Table 1). The hydrolytic reactions of $3 \mathrm{a}$ in the ether-type solvents were rapid (conv. 52\%, 51\%, and 54\% after $10 \mathrm{~min}, E=59,113$, and 27, entries 1,2, and 4), while, in EtOAc, the reaction proceeded relatively slowly with low enantioselectivity (conv. 11\%, after $10 \mathrm{~min}, E=3$, entry 3). On the basis of our earlier results [33], the reaction was also performed under solvent-free conditions when, in harmony with our earlier observation, a reasonable enantioselectivity ( $E$ 74) was observed in addition to a rapid transformation (conv. 49\% after $10 \mathrm{~min}$, entry 5). For the reason of economy (taking into account that 2-Me-THF is the most expensive selected solvent), despite the highest $E$ (113), $i \mathrm{Pr}_{2} \mathrm{O}$, with a slightly lower $E$ (108), but as significantly less expensive was identified as the most suitable solvent. 
Table 1. Solvent screening in the hydrolysis of $( \pm)-3 \mathbf{a}^{\mathrm{a}}$.

\begin{tabular}{|c|c|c|c|c|c|}
\hline Entry & Solvent (1 mL) & $e e_{\mathrm{s}}(\%)^{\mathrm{b}}$ & $e e_{\mathrm{p}}(\%)^{\mathrm{c}}$ & Conv. $(\%)^{d}$ & $E^{\mathrm{e}}$ \\
\hline 1 & TBME & 95 & 88 & 52 & 59 \\
\hline 2 & 2-Me-THF & 97 & 93 & 51 & 113 \\
\hline 3 & EtOAc & 6 & 52 & 11 & 3 \\
\hline 4 & Propylene carbonate & 92 & 79 & 54 & 27 \\
\hline 5 & no solvent & 90 & 92 & 49 & 74 \\
\hline
\end{tabular}

a $0.025 \mathrm{M}$ substrate, $30 \mathrm{mg} \mathrm{mL}^{-1}$ lipase PSIM, 5 equiv. $\mathrm{Et}_{3} \mathrm{~N}, 0.5$ equiv. $\mathrm{H}_{2} \mathrm{O}$, at $45^{\circ} \mathrm{C}$ after $10 \mathrm{~min} .{ }^{\mathrm{b}}$ according to GC after derivatization. ${ }^{\mathrm{c}}$ according to GC after double derivatization [34]. ${ }^{\mathrm{d}} c=e e_{\mathrm{s}} /\left(e e_{\mathrm{s}}+e e_{\mathrm{p}}\right)[35] .{ }^{\mathrm{e}} E=\{\ln [(1-c) \times(1$ $\left.\left.\left.-e e_{\mathrm{p}}\right)\right] / \ln \left[(1-c) \times\left(1+e e_{\mathrm{p}}\right)\right]\right\}$

Table 2. Enzyme screening in the hydrolysis of $( \pm)-3 \mathbf{a}^{\mathrm{a}}$.

\begin{tabular}{cccccc}
\hline Entry & Enzyme & $\boldsymbol{e e}_{\mathbf{s}} \mathbf{( \% )}^{\mathbf{b}}$ & $\boldsymbol{e} e_{\mathbf{p}} \mathbf{( \% )}^{\mathbf{c}}$ & Conv. $(\mathbf{\%})^{\mathbf{d}}$ & $\boldsymbol{E}^{\mathbf{e}}$ \\
\hline 1 & Lipase PSIM & 88 & 95 & 48 & 108 \\
2 & Lipase AY & 2 & 9 & 18 & 1 \\
3 & Lipase AK & 18 & 75 & 19 & 8 \\
4 & PPL & 2 & 29 & 5 & 2 \\
5 & CAL-B & 2 & 5 & 30 & 1 \\
\hline
\end{tabular}

a $0.025 \mathrm{M}$ substrate, $30 \mathrm{mg} \mathrm{mL}^{-1}$ lipase, $1 \mathrm{~mL} i \operatorname{Pr}_{2} \mathrm{O}, 5$ equiv. $\mathrm{Et}_{3} \mathrm{~N}, 0.5$ equiv. $\mathrm{H}_{2} \mathrm{O}$, at $45^{\circ} \mathrm{C}$ after $10 \mathrm{~min} .{ }^{\mathrm{b}}$ according to GC after derivatization. ${ }^{\mathrm{c}}$ according to GC after double derivatization [34]. ${ }^{\mathrm{d}} \mathrm{c}=e e_{\mathrm{s}} /\left(e e_{\mathrm{S}}+e e_{\mathrm{p}}\right)$ [35]. ${ }^{\mathrm{e}} E=\{\ln [(1-$ c) $\left.\left.\times\left(1-e e_{\mathrm{p}}\right)\right] / \ln \left[(1-c) \times\left(1+e e_{\mathrm{p}}\right)\right]\right\}$.

In order to follow up the progress of the reaction while maintaining high enantioselectivity, it was wise to slow down the reaction. When the reaction temperature decreased from $45^{\circ} \mathrm{C}$ to $25^{\circ} \mathrm{C}$, both the reaction rate and enantio-selectivity for the hydrolysis of 3a clearly decreased ( $30 \%$ conv. in $10 \mathrm{~min}$, $E=48$ vs. $48 \%$ conv. in $10 \mathrm{~min}, E=108$, Table 2, entry 1 ). To our surprise, the fastest reaction was achieved at $3{ }^{\circ} \mathrm{C}$ with the highest degree of conversion (50\% in $10 \mathrm{~min}$ ) and an $E$ value of 134 . In order to collect more information, we decided to carry out the reaction with different enzyme concentrations at $3{ }^{\circ} \mathrm{C}$. As shown in Table 3, there was no significant difference in the reaction rates, when the enzyme concentration decreased from 10 to 5 or $2 \mathrm{mg} \mathrm{mL}^{-1}$ ( $\sim 50 \%$ conv. in 10 min reaction time, entries $\left.1-3\right)$. In contrast, $E$ dropped significantly when the reaction was performed with a $2-\mathrm{mg} \mathrm{mL}^{-1}$ enzyme (entry 3). Since both high $E$ and satisfactory reaction rate were attained at $45^{\circ} \mathrm{C}$, we decided to use this optimal reaction temperature for preparative-scale reactions. Additionally, a set of preliminary experiments was performed in order to determine the influence of enzyme concentration on the reaction rate (Table 4). The reaction rate for the hydrolysis of $( \pm)$-3a clearly increased as the concentration of enzymes was increased. The highest reaction rate was observed with a $40-\mathrm{mg} \mathrm{mL}^{-1}$ enzyme (entry 5). However, for a satisfactory reaction time (the time needed to reach $50 \%$ conversion), the use of a $30-\mathrm{mg}$ $\mathrm{mL}^{-1}$ enzyme (Table 2, entry 1) was selected for preparative-scale resolutions.

Table 3. Effect of enzyme concentration in the hydrolysis of $( \pm)-3 \mathbf{a}^{\mathrm{a}}$.

\begin{tabular}{|c|c|c|c|c|c|}
\hline Entry & $\begin{array}{l}\text { Lipase PSIM } \\
\left(\mathrm{mg} \mathrm{mL} \mathrm{m}^{-1}\right)\end{array}$ & $e e_{\mathrm{s}}(\%)^{\mathrm{b}}$ & $e e_{\mathrm{p}}(\%)^{\mathrm{c}}$ & Conv. $(\%)^{d}$ & $E^{\mathrm{e}}$ \\
\hline 1 & 10 & 97 & 97 & 50 & $>200$ \\
\hline 2 & 5 & 95 & 98 & 49 & $>200$ \\
\hline 3 & 2 & 85 & 92 & 48 & 63 \\
\hline
\end{tabular}

a $0.025 \mathrm{M}$ substrate, $1 \mathrm{~mL} i \mathrm{Pr}_{2} \mathrm{O}, 5$ equiv. $\mathrm{Et}_{3} \mathrm{~N}, 0.5$ equiv. $\mathrm{H}_{2} \mathrm{O}$, at $3^{\circ} \mathrm{C}$ after $10 \mathrm{~min} .{ }^{\mathrm{b}}$ according to $\mathrm{GC}$ after derivatization. ${ }^{c}$ according to GC after double derivatization [34]. ${ }^{\mathrm{d}} c=e e_{\mathrm{s}} /\left(e e_{\mathrm{s}}+e e_{\mathrm{p}}\right)[35] .{ }^{\mathrm{e}} E=\{\ln [(1-c) \times(1-$ $\left.\left.\left.e e_{\mathrm{p}}\right)\right] / \ln \left[(1-c) \times\left(1+e e_{\mathrm{p}}\right)\right]\right\}$. 
Table 4. Effect of enzyme concentration at $45^{\circ} \mathrm{C}$ in the hydrolysis of $( \pm)-3 \mathbf{a}^{\text {a }}$

\begin{tabular}{|c|c|c|c|c|c|}
\hline Entry & $\begin{array}{l}\text { Enzyme Conc. } \\
\quad\left(\mathrm{mg} \mathrm{mL}^{-1}\right)\end{array}$ & $e e_{\mathrm{s}}(\%)^{\mathrm{b}}$ & $e e_{\mathrm{p}}(\%)^{\mathrm{c}}$ & Conv. $(\%)^{d}$ & $E^{\mathrm{e}}$ \\
\hline 1 & 2 & 4 & 81 & 5 & 10 \\
\hline 2 & 5 & 15 & 85 & 15 & 14 \\
\hline 3 & 10 & 21 & 86 & 20 & 17 \\
\hline 4 & 20 & 46 & 92 & 33 & 38 \\
\hline 5 & 40 & 97 & 89 & 52 & 74 \\
\hline
\end{tabular}

a $0.025 \mathrm{M}$ substrate, lipase PSIM, 5 equiv. $\mathrm{Et}_{3} \mathrm{~N}, 0.5$ equiv. $\mathrm{H}_{2} \mathrm{O}$, after $10 \mathrm{~min} .{ }^{\mathrm{b}}$ according to GC after derivatization.

${ }^{c}$ according to GC after double derivatization [34]. ${ }^{\mathrm{d}} c=e e_{\mathrm{s}} /\left(e e_{\mathrm{s}}+e e_{\mathrm{p}}\right)[35] .{ }^{\mathrm{e}} E=\left\{\ln \left[(1-c) \times\left(1-e e_{\mathrm{p}}\right)\right] / \ln [(1-c) \times(1\right.$

$\left.\left.\left.+e e_{\mathrm{p}}\right)\right]\right\}$.

\subsubsection{Preparative-Scale Resolutions of $( \pm)-3 \mathbf{a}-\mathbf{e}$}

Preparative-scale resolution of $( \pm)$-3a-e were performed under the optimized conditions (30 mg mL ${ }^{-1}$ lipase PSIM, 0.5 equiv $\mathrm{Et}_{3} \mathrm{~N}, 0.5$ equiv $\mathrm{H}_{2} \mathrm{O}, i \operatorname{Pr}_{2} \mathrm{O}, 45{ }^{\circ} \mathrm{C}$ ) to yield the unreacted $\beta$-amino carboxylic ester enantiomers ( $R$ )-4a-e and product $\beta$-amino acids (S)-5a-e with excellent $e e$ $(\geq 99 \%)$ and good yields ( $>48 \%$ ) (Table 5 ).

Table 5. Lipase PSIM-catalyzed hydrolysis of $( \pm)-3 \mathbf{a}-\mathbf{e}^{\mathrm{a}}$.

\begin{tabular}{|c|c|c|c|c|c|c|c|c|c|c|c|}
\hline \multirow[b]{2}{*}{ Substrate } & \multirow[b]{2}{*}{ Rt (h) } & \multirow[b]{2}{*}{ Conv. $(\%)$} & \multirow[b]{2}{*}{$E$} & \multicolumn{4}{|c|}{$\beta$-Amino Acid (5a-e) } & \multicolumn{4}{|c|}{$\beta$-Amino Ester (4a-e) } \\
\hline & & & & $\begin{array}{c}\text { Yield } \\
(\%)\end{array}$ & Isomer & $\begin{array}{l}e e^{\mathrm{b}} \\
(\%)\end{array}$ & $\begin{array}{c}{[\alpha]_{\mathrm{D}}^{25}} \\
\left(\mathrm{H}_{2} \mathrm{O}\right)\end{array}$ & $\begin{array}{c}\text { Yield } \\
(\%)\end{array}$ & Isomer & $\begin{array}{l}e e^{\mathrm{c}} \\
(\%)\end{array}$ & $\begin{array}{c}{[\alpha]_{\mathrm{D}}^{25}} \\
\left(\mathrm{CHCl}_{3}\right)\end{array}$ \\
\hline $3 a$ & 8 & 50 & $>200$ & 48 & (S) & $>99$ & $-3.1^{\mathrm{d}}$ & 49 & $(R)$ & 97 & $+17.9^{\mathrm{e}}$ \\
\hline $3 b$ & 72 & 49 & $>200$ & 48 & (S) & $>99$ & $-5^{f}$ & 38 & $(R)$ & 94 & $+9 \mathrm{~g}$ \\
\hline $3 c$ & 18 & 50 & $>200$ & 49 & (S) & $>99$ & $-3^{h}$ & 49 & $(R)$ & $>99$ & $+18.9^{\mathrm{i}}$ \\
\hline $3 d$ & 26 & 49 & $>200$ & 49 & (S) & $>99$ & $-11^{j}$ & 48 & $(R)$ & $>99$ & $+20.3^{k}$ \\
\hline $3 e$ & 23 & 50 & $>200$ & 48 & $(S)$ & $>99$ & $-13^{1}$ & 47 & $(R)$ & $>99$ & $+16^{\mathrm{m}}$ \\
\hline
\end{tabular}

a $30 \mathrm{mg} \mathrm{mL}^{-1}$ enzyme in $i \mathrm{Pr}_{2} \mathrm{O}, 5$ equiv. $\mathrm{Et}_{3} \mathrm{~N}, 0.5$ equiv. $\mathrm{H}_{2} \mathrm{O}$, at $45^{\circ} \mathrm{C}$. ${ }^{\mathrm{b}}$ according to $\mathrm{GC}$ after derivatization.

${ }^{\mathrm{c}}$ according to GC after double derivatization [34]. ${ }^{\mathrm{d}} \mathcal{c}=0.28 .{ }^{\mathrm{e}} \mathcal{c}=0.44 .{ }^{\mathrm{f}} c=0.26 .{ }^{\mathrm{g}} \mathcal{c}=0.29 .{ }^{\mathrm{h}} c=0.28 .{ }^{\mathrm{i}} c=0.41$.

${ }^{\mathrm{j}} c=0.19(\mathrm{MeOH}) .{ }^{\mathrm{k}} c=0.53 .{ }^{1} c=0.21(\mathrm{MeOH}) .{ }^{\mathrm{m}} \mathrm{c}=0.13$.

\subsubsection{Determination of Absolute Configurations}

The absolute configurations in the cases of $(S)-5 \mathbf{a}\left\{[\alpha]=-3.1\left(c 0.28, \mathrm{H}_{2} \mathrm{O}\right)\right.$, lit. $[36][\alpha]=-3.9(c 1.0$, $\left.\left.\mathrm{H}_{2} \mathrm{O}\right)\right\},(S)-5 \mathbf{b}\left\{[\alpha]=-5.0\left(c 0.26, \mathrm{H}_{2} \mathrm{O}\right)\right.$, lit. $\left.[36][\alpha]=-4.5\left(c 0.50, \mathrm{H}_{2} \mathrm{O}\right)\right\},(S)-5 \mathrm{c}\left\{[\alpha]=-3.0\left(c 0.28, \mathrm{H}_{2} \mathrm{O}\right)\right.$, lit. [37] $[\alpha]=+3.9\left(c 0.40, \mathrm{H}_{2} \mathrm{O}\right.$ for the antipode $\left.\left.(R)\right)\right\},(R)-4 \mathbf{a}\left\{[\alpha]=+17.9\left(c 0.44, \mathrm{CHCl}_{3}\right)\right.$, lit. [36] $[\alpha]=$ $-21.5\left(c\right.$ 1.0, $\mathrm{CHCl}_{3}$ for antipode $\left.\left.(S)\right)\right\},(R)-4 \mathbf{b}\left\{[\alpha]=+9.0\left(c \quad 0.29, \mathrm{CHCl}_{3}\right)\right.$, lit. [36] $[\alpha]=-12.6(c$ 0.50, $\mathrm{CHCl}_{3}$ for antipode $\left.\left.(S)\right)\right\}$ and $(R)-4 \mathrm{c}\left\{[\alpha]=+18.9\left(c 0.41, \mathrm{CHCl}_{3}\right)\right.$, lit. $\left.[18][\alpha]=+18.5\left(c 1.0, \mathrm{CHCl}_{3}\right)\right\}$ were assigned by comparing the $[\alpha]$ values with literature data. Taking into consideration the most stable conformation of the 3-amino-3-phenylpropanoate core matching nicely, the $(S)$-configuration of the products [18] and the GC chromatograms analyzed, the same enantio-preference for the (S)-selective hydrolysis by Lipase PSIM for $\mathbf{5 d}$ and $\mathbf{5 e}$ was indicated.

\section{Experimental Section}

\subsection{General Methods}

Lipase PSIM and lipase AK were from Amano Pharmaceuticals and lipase AY was from Fluka. PPL and CAL-B immobilized on acrylic resin were purchased from Sigma (Budapest, Hungary). Substituted benzaldehydes were from Sigma-Aldrich. Triethylamine was from Merck. Solvents of the highest analytical grade were from Sigma-Aldrich. Optical rotations were measured with a Perkin-Elmer 341 Polarimeter. ${ }^{1} \mathrm{H}-\mathrm{NMR}$ spectra were recorded on a Bruker Avance DRX 500 spectrometer. Melting points were determined on a Kofler apparatus (see the Supplementary Materials). The enantiomeric excess $e e$ values for the unreacted $\beta$-amino carboxylic ester and the $\beta$-amino acid enantiomers produced 
were determined by GC equipped with a Chirasil-L-Val column after double derivatization [34] with (i) diazomethane [Caution: the derivatization with diazomethane should be performed under a well-working hood] and (ii) acetic anhydride in the presence of 4-dimethylaminopyridine and pyridine [ $90^{\circ} \mathrm{C}$ for $10 \mathrm{~min} \rightarrow 170{ }^{\circ} \mathrm{C}$ (temperature rise $20^{\circ} \mathrm{C} \mathrm{min}^{-1}$ ), 10 psi]. Retention times (min) for 4a: 36.308 (antipode: 36.535), for 5a: 32.365 (antipode: 31.515), for $\mathbf{4 b}: 32.137$ (antipode: 32.550), for $\mathbf{5 b}: 29.031$ (antipode: 28.282), for 4c: 33.305 (antipode: 33.860), for 5c: 29.905 (antipode: 29.528), for 4d: 26.064 (antipode: 26.187), for 5d: 23.766 (antipode: 23.463), for 4e: 35.421 (antipode: 36.018), for 5e: 30.946 (antipode: 30.541)].

\subsection{General Procedure for the Syntheses of Racemic $\beta$-Amino Acids 2a-e}

Compounds 2a-e were synthesized based on the modified Rodionov synthesis [31,32] through condensation of the corresponding aldehydes 1a-e ( $2 \mathrm{~g})$ with malonic acid (1 equiv) in the presence of $\mathrm{NH}_{4} \mathrm{OAc}$ (2 equiv) in EtOH under reflux for $8 \mathrm{~h}$ [19]. The resulting white crystals were filtered off and washed with acetone and then they were recrystallized from $\mathrm{H}_{2} \mathrm{O}$ and acetone.

\subsection{1. ( \pm )-3-Amino-3-(3,4-Difluorophenyl) Propionic Acid 2a}

Yield: $(1.2 \mathrm{~g}, 42 \%), \mathrm{mp} 233-235^{\circ} \mathrm{C} .{ }^{1} \mathrm{H}-\mathrm{NMR}\left(\mathrm{D}_{2} \mathrm{O}, 500 \mathrm{MHz}\right): \delta=7.30-7.26(\mathrm{~m}, 1 \mathrm{H}, \mathrm{Ar}), 7.25-7.21$ $(\mathrm{m}, 1 \mathrm{H}, \mathrm{Ar}), 7.15-7.13(\mathrm{~m}, 1 \mathrm{H}, \mathrm{Ar}), 4.54(\mathrm{t}, J=7.33 \mathrm{~Hz}, 1 \mathrm{H}, \mathrm{CH}), 2.78\left(\mathrm{dd}, J_{\mathrm{AB}}=16.31 \mathrm{~Hz}, J_{\mathrm{AX}}=7.89 \mathrm{~Hz}\right.$, $\left.1 \mathrm{H}, \mathrm{C}(2) \mathrm{H}_{\mathrm{A}}\right), 2.70\left(\mathrm{dd}, J_{\mathrm{BA}}=16.27 \mathrm{~Hz}, J_{\mathrm{BX}}=6.78 \mathrm{~Hz}, 1 \mathrm{H}, \mathrm{C}(2) \mathrm{H}_{\mathrm{B}}\right)$.

\subsection{2. ( \pm )-3-Amino-3-(3,5-Difluorophenyl) Propionic Acid 2b}

Yield: (1.36 g, 48\%), mp 237-239 ${ }^{\circ} \mathrm{C} .{ }^{1} \mathrm{H}-\mathrm{NMR}\left(\mathrm{D}_{2} \mathrm{O}, 500 \mathrm{MHz}\right): \delta=6.98-6.97(\mathrm{~m}, 2 \mathrm{H}, \mathrm{Ar}), 6.93-6.89$ $(\mathrm{m}, 1 \mathrm{H}, \mathrm{Ar}), 4.56(\mathrm{t}, J=7.23 \mathrm{~Hz}, 1 \mathrm{H}, \mathrm{CH}), 2.78\left(\mathrm{dd}, J_{\mathrm{AB}}=16.34 \mathrm{~Hz}, J_{\mathrm{AX}}=7.84 \mathrm{~Hz}, 1 \mathrm{H}, \mathrm{C}(2) \mathrm{H}_{\mathrm{A}}\right), 2.71(\mathrm{dd}$, $\left.J_{\mathrm{BA}}=16.34 \mathrm{~Hz}, J_{\mathrm{BX}}=6.61 \mathrm{~Hz}, 1 \mathrm{H}, \mathrm{C}(2) \mathrm{H}_{\mathrm{B}}\right)$.

\subsection{3. ( \pm )-3-Amino-3-(4-Fluorophenyl) Propionic Acid 2c}

Yield: $(1.50 \mathrm{~g}, 51 \%), \mathrm{mp} 244-246{ }^{\circ} \mathrm{C} .{ }^{1} \mathrm{H}-\mathrm{NMR}\left(\mathrm{D}_{2} \mathrm{O}, 500 \mathrm{MHz}\right): \delta=7.37-7.34(\mathrm{~m}, 2 \mathrm{H}, \mathrm{Ar}), 7.11-7.07$ $(\mathrm{m}, 2 \mathrm{H}, \mathrm{Ar}), 4.54(\mathrm{t}, J=7.31 \mathrm{~Hz}, 1 \mathrm{H}, \mathrm{CH}), 2.81\left(\mathrm{dd}, J_{\mathrm{AB}}=16.10 \mathrm{~Hz}, J_{\mathrm{AX}}=8.05 \mathrm{~Hz}, 1 \mathrm{H}, \mathrm{C}(2) \mathrm{H}_{\mathrm{A}}\right), 2.72(\mathrm{dd}$, $\left.J_{\mathrm{BA}}=16.10 \mathrm{~Hz}, J_{\mathrm{BX}}=6.73 \mathrm{~Hz}, 1 \mathrm{H}, \mathrm{C}(2) \mathrm{H}_{\mathrm{B}}\right)$.

\subsection{4. ( \pm )-3-Amino-3-(2-Fluoro-4-Triflouromethylphenyl) Propionic Acid 2d}

Yield: (0.786 g, 30\%), mp 251-253 ${ }^{\circ} \mathrm{C} .{ }^{1} \mathrm{H}-\mathrm{NMR}\left(\mathrm{D}_{2} \mathrm{O}, 500 \mathrm{MHz}\right): \delta=7.75-7.72(\mathrm{~m}, 1 \mathrm{H}, \mathrm{Ar})$, $7.62-7.57(\mathrm{~m}, 2 \mathrm{H}, \mathrm{Ar}), 4.89(\mathrm{t}, J=7 \mathrm{~Hz}, 1 \mathrm{H}, \mathrm{CH}), 2.82\left(\mathrm{dd}, J_{\mathrm{AB}}=16.63 \mathrm{~Hz}, J_{\mathrm{AX}}=8.63 \mathrm{~Hz}, 1 \mathrm{H}, \mathrm{C}(2) \mathrm{H}_{\mathrm{A}}\right)$, $2.77\left(\mathrm{dd}, J_{\mathrm{BA}}=16.86 \mathrm{~Hz}, J_{\mathrm{BX}}=5.31 \mathrm{~Hz}, 1 \mathrm{H}, \mathrm{C}(2) \mathrm{H}_{\mathrm{B}}\right)$.

\subsection{5. ( \pm )-3-Amino-3-(2-Fluoro-4-Methylphenyl) Propionic Acid 2e}

Yield: (0.4275 g, 15\%), mp 239-241 ${ }^{\circ} \mathrm{C} .{ }^{1} \mathrm{H}-\mathrm{NMR}\left(\mathrm{D}_{2} \mathrm{O}, 500 \mathrm{MHz}\right): \delta=7.39-7.36(\mathrm{~m}, 1 \mathrm{H}, \mathrm{Ar})$, 7.11-7.03 (m, 2H, Ar), $4.84(\mathrm{t}, J=7.78 \mathrm{~Hz}, 1 \mathrm{H}, \mathrm{CH}), 2.80\left(\mathrm{dd}, J_{\mathrm{AB}}=16.81 \mathrm{~Hz}, J_{\mathrm{AX}}=9.82 \mathrm{~Hz}, 1 \mathrm{H}, \mathrm{C}(2) \mathrm{H}_{\mathrm{A}}\right)$, $2.69\left(\mathrm{dd}, \mathrm{J}_{\mathrm{BA}}=16.75 \mathrm{~Hz}, \mathrm{~J}_{\mathrm{BX}}=4.24 \mathrm{~Hz}, 1 \mathrm{H}, \mathrm{C}(2) \mathrm{H}_{\mathrm{B}}\right), 2.38\left(\mathrm{~s}, 3 \mathrm{H}, \mathrm{CH}_{3}\right)$.

\subsection{General Procedure for the Syntheses of Racemic $\beta$-Amino Carboxylic Ester Hydrochloride Salts 3a-e}

$\mathrm{SOCl}_{2}$ (1.05 equiv) was added to $30 \mathrm{~mL}$ of $\mathrm{EtOH}$ at a temperature kept under $-15^{\circ} \mathrm{C}$ with saline ice. To this solution, $2 \mathrm{a}-\mathbf{e}(1 \mathrm{~g})$ were added at once. The mixture was stirred at $0{ }^{\circ} \mathrm{C}$ for $30 \mathrm{~min}$, then at room temperature for $3 \mathrm{~h}$, and, finally, heated under reflux for $1 \mathrm{~h}$. The solvent was evaporated off under

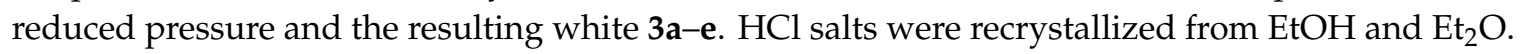




\subsubsection{Hydrochloride Salt of Ethyl ( \pm )-3-Amino-3-(3,4-Difluorophenyl) Propanoate 3a. $\mathrm{HCl}$}

Yield: $(1 \mathrm{~g}, 76 \%), \mathrm{mp} 142-144{ }^{\circ} \mathrm{C} .{ }^{1} \mathrm{H}-\mathrm{NMR}\left(\mathrm{D}_{2} \mathrm{O}, 500 \mathrm{MHz}\right): \delta=7.32-7.28(\mathrm{~m}, 1 \mathrm{H}, \mathrm{Ar}), 7.27-7.23$ (m, 1H, Ar), 7.18-7.16 (m, 1H, Ar), $4.69(\mathrm{t}, J=8.73 \mathrm{~Hz}, 1 \mathrm{H}, \mathrm{CH}), 4.05-4.01\left(\mathrm{~m}, 2 \mathrm{H}, \mathrm{CH}_{2}\right), 3.08(\mathrm{dd}$, $\left.J_{\mathrm{AB}}=16.77 \mathrm{~Hz}, J_{\mathrm{AX}}=7.20 \mathrm{~Hz}, 1 \mathrm{H}, \mathrm{C}(2) \mathrm{H}_{\mathrm{A}}\right), 3.00\left(\mathrm{dd}, J_{\mathrm{BA}}=16.77 \mathrm{~Hz}, J_{\mathrm{BX}}=7.50 \mathrm{~Hz}, 1 \mathrm{H}, \mathrm{C}(2) \mathrm{H}_{\mathrm{B}}\right), 1.05(\mathrm{t}$, $\left.J=7.09 \mathrm{~Hz}, 3 \mathrm{H}, \mathrm{CH}_{3}\right) .{ }^{13} \mathrm{C}-\mathrm{NMR}\left(\mathrm{D}_{2} \mathrm{O}, 126 \mathrm{MHz}\right): \delta=13.2,37.9,50.7,62.5,116.6\left(\mathrm{~d},{ }^{2} J_{\mathrm{C}-\mathrm{F}}=18.42 \mathrm{~Hz}\right)$, $118.4\left(\mathrm{~d},{ }^{2} J_{\mathrm{C}-\mathrm{F}}=17.65 \mathrm{~Hz}\right), 124.1\left(\mathrm{dd},{ }^{3} J_{\mathrm{C}-\mathrm{F}}=7.10 \mathrm{~Hz},{ }^{4} J_{\mathrm{C}-\mathrm{F}}=3.64 \mathrm{~Hz}\right), 132.2\left(\mathrm{~d},{ }^{3} J_{\mathrm{C}-\mathrm{F}}=3.87 \mathrm{~Hz}\right)$, $150.2\left(\mathrm{dd},{ }^{1} J_{\mathrm{C}-\mathrm{F}}=250.46 \mathrm{~Hz},{ }^{2} J_{\mathrm{C}-\mathrm{F}}=16.46 \mathrm{~Hz}\right), 150.6\left(\mathrm{dd},{ }^{1} J_{\mathrm{C}-\mathrm{F}}=241.81 \mathrm{~Hz},{ }^{2} J_{\mathrm{C}-\mathrm{F}}=5.74 \mathrm{~Hz}\right), 171.3$. ${ }^{19} \mathrm{~F}-\mathrm{NMR}\left(\mathrm{D}_{2} \mathrm{O}, 471 \mathrm{MHz}\right): \delta=-136.6 \mathrm{~Hz},-136.9 \mathrm{~Hz}$.

\subsubsection{Hydrochloride Salt of Ethyl ( \pm )-3-Amino-3-(3,5-Difluorophenyl) Propanoate 3b. $\mathrm{HCl}$}

Yield: $(1.15 \mathrm{~g}, 87 \%), \mathrm{mp} 182-184{ }^{\circ} \mathrm{C} .{ }^{1} \mathrm{H}-\mathrm{NMR}\left(\mathrm{D}_{2} \mathrm{O}, 500 \mathrm{MHz}\right): \delta=7.02-7.01(\mathrm{~m}, 2 \mathrm{H}, \mathrm{Ar}), 6.97-6.93$ $(\mathrm{m}, 1 \mathrm{H}, \mathrm{Ar}), 4.74(\mathrm{t}, J=7.20 \mathrm{~Hz}, 1 \mathrm{H}, \mathrm{CH}), 4.07-4.02\left(\mathrm{~m}, 2 \mathrm{H}, \mathrm{CH}_{2}\right), 3.11\left(\mathrm{dd}, J_{\mathrm{AB}}=16.98 \mathrm{~Hz}, J_{\mathrm{AX}}=7.24 \mathrm{~Hz}\right.$, $\left.1 \mathrm{H}, \mathrm{C}(2) \mathrm{H}_{\mathrm{A}}\right), 2.81\left(\mathrm{dd}, J_{\mathrm{BA}}=16.96 \mathrm{~Hz}, J_{\mathrm{BX}}=7.23 \mathrm{~Hz}, 1 \mathrm{H}, \mathrm{C}(2) \mathrm{H}_{\mathrm{B}}\right), 1.08\left(\mathrm{t}, J=6.98 \mathrm{~Hz}, 3 \mathrm{H}, \mathrm{CH}_{3}\right)$. ${ }^{13} \mathrm{C}-\mathrm{NMR}\left(\mathrm{D}_{2} \mathrm{O}, 126 \mathrm{MHz}\right): \delta=15.6,40.2,53.2,65.0,107.6\left(\mathrm{t},{ }^{2} J_{\mathrm{C}-\mathrm{F}}=25.43 \mathrm{~Hz}\right), 113.0\left(\mathrm{dd},{ }^{2} J_{\mathrm{C}-\mathrm{F}}=20.28 \mathrm{~Hz}\right.$, $\left.{ }^{4} J_{\mathrm{C}-\mathrm{F}}=6.70 \mathrm{~Hz}\right), 141.0\left(\mathrm{t},{ }^{3} J_{\mathrm{C}-\mathrm{F}}=9.47 \mathrm{~Hz}\right), 165.6\left(\mathrm{dd},{ }^{1} J_{\mathrm{C}-\mathrm{F}}=248.20 \mathrm{~Hz},{ }^{3} J_{\mathrm{C}-\mathrm{F}}=13.04 \mathrm{~Hz}\right), 173.7 .{ }^{19} \mathrm{~F}-\mathrm{NMR}$ $\left(\mathrm{D}_{2} \mathrm{O}, 471 \mathrm{MHz}\right): \delta=-108.3 \mathrm{~Hz}$.

\subsubsection{Hydrochloride Salt of Ethyl ( \pm )-3-Amino-3-(4-Flourophenyl) Propanoate 3c. HCl}

Yield: $(1.11 \mathrm{~g}, 82 \%)$, mp $165-167{ }^{\circ} \mathrm{C} .{ }^{1} \mathrm{H}-\mathrm{NMR}\left(\mathrm{D}_{2} \mathrm{O}, 500 \mathrm{MHz}\right): \delta=7.39-7.37(\mathrm{~m}, 2 \mathrm{H}, \mathrm{Ar}), 7.13-7.09$ $(\mathrm{m}, 2 \mathrm{H}, \mathrm{Ar}), 4.70(\mathrm{t}, J=7.82 \mathrm{~Hz}, 1 \mathrm{H}, \mathrm{CH}), 4.04-3.99\left(\mathrm{~m}, 2 \mathrm{H}, \mathrm{CH}_{2}\right), 3.09\left(\mathrm{dd}, J_{\mathrm{AB}}=16.61 \mathrm{~Hz}, J_{\mathrm{AX}}=7.17 \mathrm{~Hz}\right.$, $\left.1 \mathrm{H}, \mathrm{C}(2) \mathrm{H}_{\mathrm{A}}\right), 3.00\left(\mathrm{dd}, J_{\mathrm{BA}}=16.66 \mathrm{~Hz}, J_{\mathrm{BX}}=7.65 \mathrm{~Hz}, 1 \mathrm{H}, \mathrm{C}(2) \mathrm{H}_{\mathrm{B}}\right), 1.05\left(\mathrm{t}, J=7.21 \mathrm{~Hz}, 3 \mathrm{H}, \mathrm{CH}_{3}\right)$. ${ }^{13} \mathrm{C}-\mathrm{NMR}\left(\mathrm{D}_{2} \mathrm{O}, 126 \mathrm{MHz}\right): \delta=13.3,38.3,51.1,62.6,116.3\left(\mathrm{~d},{ }^{2} J_{\mathrm{C}-\mathrm{F}}=22.08 \mathrm{~Hz}\right), 129.5\left(\mathrm{~d},{ }^{3} J_{\mathrm{C}-\mathrm{F}}=8.94 \mathrm{~Hz}\right)$, $131.3\left(\mathrm{~d},{ }^{4} J_{\mathrm{C}-\mathrm{F}}=2.99 \mathrm{~Hz}\right), 163.2\left(\mathrm{~d},{ }^{1} J_{\mathrm{C}-\mathrm{F}}=246.30 \mathrm{~Hz}\right), 171.6 .{ }^{19} \mathrm{~F}-\mathrm{NMR}\left(\mathrm{D}_{2} \mathrm{O}, 471 \mathrm{MHz}\right): \delta=-112.3 \mathrm{~Hz}$.

3.3.4. Hydrochloride Salt of Ethyl ( \pm )-3-Amino-3-(2-Fluoro-4-Trifluoromethylphenyl) Propanoate $3 \mathbf{d}$. HCl

Yield: $(1.2 \mathrm{~g}, 95 \%), \mathrm{mp} 133-135{ }^{\circ} \mathrm{C} .{ }^{1} \mathrm{H}-\mathrm{NMR}\left(\mathrm{D}_{2} \mathrm{O}, 500 \mathrm{MHz}\right): \delta=7.61-7.58(\mathrm{~m}, 1 \mathrm{H}, \mathrm{Ar})$, 7.56-7.53 (m, 2H, Ar), $5.03(\mathrm{t}, J=7.31 \mathrm{~Hz}, 1 \mathrm{H}, \mathrm{CH}), 4.07-4.02\left(\mathrm{~m}, 2 \mathrm{H}, \mathrm{CH}_{2}\right), 3.20\left(\mathrm{dd}, J_{\mathrm{AB}}=16.90 \mathrm{~Hz}\right.$, $\left.J_{\mathrm{AX}}=7.27 \mathrm{~Hz}, 1 \mathrm{H}, \mathrm{C}(2) \mathrm{H}_{\mathrm{A}}\right), 3.12\left(\mathrm{dd}, J_{\mathrm{BA}}=16.94 \mathrm{~Hz}, J_{\mathrm{BX}}=7.35 \mathrm{~Hz}, 1 \mathrm{H}, \mathrm{C}(2) \mathrm{H}_{\mathrm{B}}\right), 1.07(\mathrm{t}, J=7.51 \mathrm{~Hz}$, $\left.3 \mathrm{H}, \mathrm{CH}_{3}\right) .{ }^{13} \mathrm{C}-\mathrm{NMR}\left(\mathrm{D}_{2} \mathrm{O}, 126 \mathrm{MHz}\right): \delta=13.1,36.9,45.8\left(\mathrm{~d},{ }^{3} J_{\mathrm{C}-\mathrm{F}}=2.69 \mathrm{~Hz}\right), 62.6,113.9(\mathrm{dq}$, $\left.{ }^{2} J_{\mathrm{C}-\mathrm{F}}=25.50 \mathrm{~Hz},{ }^{3} J_{\mathrm{C}-\mathrm{F}}=3.80 \mathrm{~Hz}\right), 123\left(\mathrm{q},{ }^{1} J_{\mathrm{C}-\mathrm{F}}=274.12 \mathrm{~Hz}\right), 122.2(\mathrm{~m}), 126.3\left(\mathrm{~d},{ }^{2} J_{\mathrm{C}-\mathrm{F}}=12.87 \mathrm{~Hz}\right)$, $129.8\left(\mathrm{~d},{ }^{3} J_{\mathrm{C}-\mathrm{F}}=3.44 \mathrm{~Hz}\right), 133.2(\mathrm{~m}), 160.0\left(\mathrm{~d},{ }^{1} J_{\mathrm{C}-\mathrm{F}}=248.95 \mathrm{~Hz}\right), 171.1 .{ }^{19} \mathrm{~F}-\mathrm{NMR}\left(\mathrm{D}_{2} \mathrm{O}, 471 \mathrm{MHz}\right):$ $\delta=-62.6 \mathrm{~Hz},-114.9 \mathrm{~Hz}$.

\subsubsection{Hydrochloride Salt of Ethyl ( \pm )-3-Amino-3-(2-Fluoro-4-Methylphenyl) Propanoate 3e. HCl}

Yield: $(1.30 \mathrm{~g}, 98 \%), \mathrm{mp} 172-174{ }^{\circ} \mathrm{C} .{ }^{1} \mathrm{H}-\mathrm{NMR}\left(\mathrm{D}_{2} \mathrm{O}, 500 \mathrm{MHz}\right): \delta=7.26-7.23(\mathrm{~m}, 1 \mathrm{H}, \mathrm{Ar}), 7.03-6.99$ $(\mathrm{m}, 2 \mathrm{H}, \mathrm{Ar}), 4.90(\mathrm{t}, J=7.51 \mathrm{~Hz}, 1 \mathrm{H}, \mathrm{CH}), 4.05-4.01\left(\mathrm{~m}, 2 \mathrm{H}, \mathrm{CH}_{2}\right), 3.15\left(\mathrm{dd}, J_{\mathrm{AB}}=16.55 \mathrm{~Hz}, J_{\mathrm{AX}}=7.32 \mathrm{~Hz}\right.$, $\left.1 \mathrm{H}, \mathrm{C}(2) \mathrm{H}_{\mathrm{A}}\right), 3.06\left(\mathrm{dd}, J_{\mathrm{BA}}=16.57 \mathrm{~Hz}, J_{\mathrm{BX}}=7.73 \mathrm{~Hz}, 1 \mathrm{H}, \mathrm{C}(2) \mathrm{H}_{\mathrm{B}}\right), 2.25\left(\mathrm{~s}, 3 \mathrm{H}, \mathrm{CH}_{3}\right), 1.06(\mathrm{t}, J=7.12 \mathrm{~Hz}$, $\left.3 \mathrm{H}, \mathrm{CH}_{3},\right) .{ }^{13} \mathrm{C}-\mathrm{NMR}\left(\mathrm{D}_{2} \mathrm{O}, 126 \mathrm{MHz}\right): \delta=13.1,20.3,37.2,46.1\left(\mathrm{~d},{ }^{3} J_{\mathrm{C}-\mathrm{F}}=2.77 \mathrm{~Hz}\right), 62.5,116.6(\mathrm{~d}$, $\left.{ }^{2} J_{\mathrm{C}-\mathrm{F}}=21.22 \mathrm{~Hz}\right), 118.8\left(\mathrm{~d},{ }^{2} J_{\mathrm{C}-\mathrm{F}}=13.22 \mathrm{~Hz},\right), 125.8\left(\mathrm{~d},{ }^{4} J_{\mathrm{C}-\mathrm{F}}=2.85 \mathrm{~Hz}\right), 128.3\left(\mathrm{~d},{ }^{3} J_{\mathrm{C}-\mathrm{F}}=3.71 \mathrm{~Hz}\right)$, $143.3\left(\mathrm{~d},{ }^{3} J_{\mathrm{C}-\mathrm{F}}=8.42 \mathrm{~Hz}\right), 160.1\left(\mathrm{~d},{ }^{1} J_{\mathrm{C}-\mathrm{F}}=245.57 \mathrm{~Hz}\right), 171.5 .{ }^{19} \mathrm{~F}-\mathrm{NMR}\left(\mathrm{D}_{2} \mathrm{O}, 471 \mathrm{MHz}\right): \delta=-118.5 \mathrm{~Hz}$.

\subsection{General Procedure for the Preparative-Scale Resolutions of ( \pm ) 3a-e}

Racemic hydrochloride salts $3 \mathbf{a}-\mathbf{e}(200 \mathrm{mg})$ were dissolved in $i \operatorname{Pr}_{2} \mathrm{O}(10 \mathrm{~mL})$. Lipase PSIM ( $30 \mathrm{mg} \mathrm{mL}^{-1}$ ), $\mathrm{Et}_{3} \mathrm{~N}$ (5 equiv), and $\mathrm{H}_{2} \mathrm{O}$ (0.5 equiv) were added and the mixture was shaken in an incubator shaker at $45^{\circ} \mathrm{C}$ for Rt: $8 \mathrm{~h} \mathrm{3a}, 72 \mathrm{~h} \mathrm{3b}, 18 \mathrm{~h} \mathrm{3c}, 26 \mathrm{~h} \mathrm{3d}, 23 \mathrm{~h} \mathrm{3e}$ (Table 5). Reactions were stopped by filtering off the enzyme at close to $50 \%$ conversion. The filtered enzyme was washed with $\mathrm{Et}_{2} \mathrm{O}(3 \times 15 \mathrm{~mL})$. The solvents were dried by using $\mathrm{Na}_{2} \mathrm{SO}_{4}$, and then evaporated off to yield unreacted $\beta$-amino carboxylic esters $(R)-4 a-e$. The filtered enzyme was washed with distilled $\mathrm{H}_{2} \mathrm{O}(3 \times 15 \mathrm{~mL})$. Then evaporation of the filtrate yielded the crystalline (S)-5a-e products, which where recrystallized 
from $\mathrm{EtOH}$ and $\mathrm{H}_{2} \mathrm{O}$. All of the enantiomers formed in enzymatic reactions were isolated in basic form due to a relatively slow in situ liberation of basic amino ester from its hydrochloric salt, which is followed by enzymatic hydrolysis.

\subsection{1. (R)-Ethyl 3-Amino-3-(3,4-Difluorophenyl) Propanoate 4a}

Yield: $(84 \mathrm{mg}, 48.7 \%),[\alpha]=+17.9\left(c 0.44, \mathrm{CHCl}_{3}\right)$, lit. [36] [ $\left.\alpha\right]=-21.5\left(c 1.0, \mathrm{CHCl}_{3}\right.$ for antipode $(S)),[\alpha]=+4.1(c 0.33, \mathrm{EtOH})$. The ${ }^{1} \mathrm{H}-\mathrm{NMR}\left(\mathrm{D}_{2} \mathrm{O}, 500 \mathrm{MHz}\right)$ spectroscopic data were similar to those for $3 a$.

\subsection{2. (R)-Ethyl 3-Amino-3-(3,5-Difluorophenyl) Propanoate 4b}

Yield: (66 mg, 38.26\%), $[\alpha]=+9.0\left(c\right.$ 0.29, $\left.\mathrm{CHCl}_{3}\right)$, lit. [36] $[\alpha]=-12.6\left(c 0.50, \mathrm{CHCl}_{3}\right.$ for antipode $(S)),[\alpha]=-5.0(c$ 0.20, EtOH $)$. The ${ }^{1} \mathrm{H}-\mathrm{NMR}\left(\mathrm{D}_{2} \mathrm{O}, 500 \mathrm{MHz}\right)$ spectroscopic data were similar to those for $3 b$.

\subsection{3. (R)-Ethyl 3-Amino-3-(4-Fluorophenyl) Propanoate 4c}

Yield: $(83 \mathrm{mg}, 49 \%),[\alpha]=+18.9\left(\right.$ c $\left.0.41, \mathrm{CHCl}_{3}\right)$, lit. $[18][\alpha]=+18.5\left(\right.$ c $\left.1.0, \mathrm{CHCl}_{3}\right),[\alpha]=+12.8$ (c $0.32, \mathrm{EtOH})$. The ${ }^{1} \mathrm{H}-\mathrm{NMR}\left(\mathrm{D}_{2} \mathrm{O}, 500 \mathrm{MHz}\right)$ spectroscopic data were similar to those for $3 \mathrm{c}$.

\subsection{4. (R)-Ethyl 3-Amino-3-(2-Fluoro-4-Triflouromethylphenyl) Propanoate 4d}

Yield: $(84.6 \mathrm{mg}, 47.83 \%),[\alpha]=+20.3\left(c 0.53, \mathrm{CHCl}_{3}\right),[\alpha]=+13.7$ (c 0.30, EtOH). The ${ }^{1} \mathrm{H}-\mathrm{NMR}$ $\left(\mathrm{D}_{2} \mathrm{O}, 500 \mathrm{MHz}\right)$ spectroscopic data were similar to those for $\mathbf{3 d}$.

\subsection{5. (R)-Ethyl 3-Amino-3-(2-Fluoro-4-Methylphenyl) Propanoate 4e}

Yield: $(94.8 \mathrm{mg}, 47.4 \%),[\alpha]=+16.0\left(c 0.13, \mathrm{CHCl}_{3}\right),[\alpha]=+6.0\left(c\right.$ 0.21, EtOH). The ${ }^{1} \mathrm{H}-\mathrm{NMR}\left(\mathrm{D}_{2} \mathrm{O}\right.$, $500 \mathrm{MHz})$ spectroscopic data were similar to those for $3 \mathbf{e}$.

\subsection{6. (S)-3-Amino-3-(3,4-Difluorophenyl) Propionic Acid 5a}

Yield: $(72.8 \mathrm{mg}, 48.08 \%),[\alpha]=-3.1\left(c \mathrm{c} 0.28, \mathrm{H}_{2} \mathrm{O}\right)$, lit. [36] $[\alpha]=-3.9\left(c 1.0, \mathrm{H}_{2} \mathrm{O}\right), \mathrm{mp} 229-231^{\circ} \mathrm{C}$, lit. [38] mp $226-230{ }^{\circ} \mathrm{C}$. The ${ }^{1} \mathrm{H}-\mathrm{NMR}\left(\mathrm{D}_{2} \mathrm{O}, 500 \mathrm{MHz}\right)$ spectroscopic data were similar to those for $\mathbf{2 a}$.

\subsection{7. (S)-3-Amino-3-(3,5-Difluorophenyl) Propionic Acid 5b}

Yield: $(73 \mathrm{mg}, 48.22 \%),[\alpha]=-5.0\left(c 0.26, \mathrm{H}_{2} \mathrm{O}\right)$, lit. [36] $[\alpha]=-4.5\left(c 0.50, \mathrm{H}_{2} \mathrm{O}\right), \mathrm{mp} 256-258^{\circ} \mathrm{C}$. The ${ }^{1} \mathrm{H}-\mathrm{NMR}\left(\mathrm{D}_{2} \mathrm{O}, 500 \mathrm{MHz}\right)$ spectroscopic data were similar to those for $\mathbf{2} \mathbf{b}$.

\subsection{8. (S)-3-Amino-3-(4-Fluorophenyl) Propionic Acid 5c}

Yield: (71.7 mg, 48.5\%), $[\alpha]=-3.0\left(c 0.28, \mathrm{H}_{2} \mathrm{O}\right)$, lit. [37] $[\alpha]=+3.9\left(c 0.40, \mathrm{H}_{2} \mathrm{O}\right.$ for antipode $\left.(R)\right)$, $\mathrm{mp} 242-244^{\circ} \mathrm{C}$, lit. [37] $\mathrm{mp} 245-247^{\circ} \mathrm{C}$. The ${ }^{1} \mathrm{H}-\mathrm{NMR}\left(\mathrm{D}_{2} \mathrm{O}, 500 \mathrm{MHz}\right)$ spectroscopic data were similar to those for $2 \mathrm{c}$.

\subsection{9. (S)-3-Amino-3-(2-Fluoro-4-Trifluoromethylphenyl) Propionic Acid 5d}

Yield: $(77.5 \mathrm{mg}, 48.7 \%),[\alpha]=-11.0(c$ 0.19, $\mathrm{MeOH}), \mathrm{mp} 255-257^{\circ} \mathrm{C}$. The ${ }^{1} \mathrm{H}-\mathrm{NMR}\left(\mathrm{D}_{2} \mathrm{O}, 500 \mathrm{MHz}\right)$ spectroscopic data were similar to those for $\mathbf{2 d}$.

\subsubsection{0. (S)-3-Amino-3-(2-Fluoro-4-Methylphenyl) Propionic Acid 5e}

Yield: $(84.8 \mathrm{mg}, 48.42 \%),[\alpha]=-13.0(c 0.21, \mathrm{MeOH}), \mathrm{mp} 258-260{ }^{\circ} \mathrm{C}$. The ${ }^{1} \mathrm{H}-\mathrm{NMR}\left(\mathrm{D}_{2} \mathrm{O}, 500 \mathrm{MHz}\right)$ spectroscopic data were similar to those for $2 \mathbf{e}$. 


\section{Conclusions}

Novel fluorine-containing amino acid enantiomers have been prepared through the hydrolysis of racemic $\beta$-amino carboxylic ester hydrochloride salts $3 \mathbf{a}-\mathbf{e}$ catalyzed by lipase PSIM. Excellent enantioselectivities $(E>200)$ were obtained when the reactions were performed with $\mathrm{H}_{2} \mathrm{O}$ as a nucleophile in $i \operatorname{Pr}_{2} \mathrm{O}$ at $45^{\circ} \mathrm{C}$, in the presence of $\mathrm{Et}_{3} \mathrm{~N}$. Both unreacted amino carboxylic esters $(R)-4 \mathbf{a}-\mathbf{e}$ and product amino acids $(S)$-5a-e were isolated with excellent $e e$ (usually $\geq 99 \%$ ) and good yields $(>48 \%)$. Suitable analytical methods were devised to follow the enzymatic reactions and calculate the enantiomeric excess, conversions, and enantio-selectivities.

Supplementary Materials: The following are available online, Figures S1-S15: ${ }^{1} \mathrm{H}-\mathrm{NMR}$ spectra for $( \pm)-2 \mathrm{a}-\mathbf{e}$, $( \pm)-\mathbf{3 a}-\mathbf{e},(R)-\mathbf{4 a}-\mathbf{e}$, and $(S)-\mathbf{5 a}-\mathbf{e}$, Figures S16-S25: GC chromatograms for $(S)-\mathbf{5 a}-\mathbf{e}$ and $(R)-\mathbf{4 a - e}$, Figures S26-S30: ${ }^{13}$ C-NMR spectra for $( \pm)-3 a-e$, Figures S31-S35: ${ }^{19} \mathrm{~F}-\mathrm{NMR}$ spectra for $( \pm)-\mathbf{3 a}-\mathbf{e}$.

Author Contributions: F.F. and E.F. planned and designed the project. S.S. performed the syntheses and characterized the synthesized compounds. E.F. and S.S. prepared the manuscript for publication. All authors discussed the results and commented on the manuscript. All authors have read and agreed to the published version of the manuscript.

Funding: The authors thank the Hungarian Scientific Research Council (OTKA, K129049) and the Ministry of National Economy, National Research, Development and Innovation Office (GINOP, 2.3.2-15-2016-00014) for financial support.

Conflicts of Interest: The authors declare no conflict of interest.

\section{References}

1. Wasserman, H.H.; Berger, G.D. The use of $\beta$-lactams in the synthesis of spermine and spermidine alkaloids. Tetrahedron 1983, 39, 2459-2464. [CrossRef]

2. Renault, O.; Guillon, J.; Dallemagne, P.; Rault, S. Efficient synthesis of 2-aryl-6-methyl-2,3-dihydro1H-pyridin-4-ones. Tetrahedron Lett. 2000, 41, 681-683. [CrossRef]

3. Juaristi, E.; Soloshonok, V.A. Enantioselective Synthesis of $\beta$-Amino Acids, 2nd ed.; Wiley: Hoboken, NJ, USA, 2005.

4. Wani, M.C.; Taylor, H.L.; Wall, M.E.; Coggon, P.; McPhail, A.T. Plant antitumore agents. VI. Isolation and structure of taxol, a novel antileukemic and antitumor agent from taxus brevifolia. J. Am. Chem. Soc. 1971, 93, 2325-2327. [CrossRef] [PubMed]

5. Jin, M.; Fischbach, M.A.; Clardy, J. A biosynthetic gene cluster for the acetyl-CoA carboxylase inhibitor andrimid. J. Am. Chem. Soc. 2006, 128, 10660-10661. [CrossRef] [PubMed]

6. Cardillo, G.; Gentilucci, L.; Melchiorre, P.; Spampinato, S. Synthesis and binding activity of endomorphin1-analogues containing $\beta$-amino acids. Bioorg. Med. Chem. Lett. 2000, 10, 2755-2758. [CrossRef]

7. Ojima, I. Fluorine in Medicinal Chemistry and Chemical Biology; Wiley-Blackwell: Chichester, UK, 2009.

8. Kirsch, P. Modern Fluoroorganic Chemistry Synthesis, Reactivity, Applications; Wiley-VCH: Weinheim, Germany, 2006.

9. Thornberry, N.; Weber, A. Discovery of JANUVIA ${ }^{\mathrm{TM}}$ (Sitagiliptin) a selective dipeptidyl peptidase IV inhibitor for the treatment of type2 diabetes. Curr. Top. Med. Chem. 2007, 7, 557-568. [CrossRef]

10. Pepin, J.; Guern, C.; Milord, F.; Schechter, P.J. Difluoromethylornithine for arseno-resistant trypanosome brucei gambiense sleeping sickness. Lancet 1987, 330, 1431-1433. [CrossRef]

11. Wolf, J.E.; Shander, D.; Huber, F.; Jackson, J.; Lin, C.-S.; Mathes, B.M.; Schrode, K. Randomized, double-blind clinical evaluation of the efficacy and safety of topical eflornithine $\mathrm{HCl} 13.9 \%$ cream in the treatment of women with facial hair: Eflornithine treatment for unwanted facial hair. Int. J. Dermatol. 2007, 46, 94-98. [CrossRef]

12. Cimarelli, C.; Palmieri, G.; Volpini, E. An improved synthesis of enantiopure $\beta$-amino acids. Synth. Commun. 2001, 31, 2943-2953. [CrossRef]

13. Sivakumar, A.V.; Babu, G.S.; Bhat, S.V. Asymmetric synthesis of $\beta$-amino acids through application of chiral sulfoxide. Tetrahedron Asymmetry 2001, 12, 1095-1099. [CrossRef]

14. Wenzel, A.G.; Jacobsen, E.N. Asymmetric catalytic mannich reactions catalyzed by urea derivatives: Enantioselective synthesis of $\beta$-aryl- $\beta$-amino acids. J. Am. Chem. Soc. 2002, 124, 12964-12965. [CrossRef] 
15. Vaidyanathan, R.; Hesmondhalgh, L.; Hu, S. A chemoenzymatic synthesis of an androgen receptor antagonist. Org. Process Res. Dev. 2007, 11, 903-906. [CrossRef]

16. Allwein, S.P.; Roemmele, R.C.; Haley, J.J.; Mowrey, D.R.; Petrillo, D.E.; Reif, J.J.; Gingrich, D.E.; Bakale, R.P. Development and scale-up of an optimized route to the ALK inhibitor CEP-28122. Org. Process Res. Dev. 2012, 16, 148-155. [CrossRef]

17. Forró, E.; Fülöp, F. Recent lipase-catalyzed hydrolytic approaches to pharmacologically important $\beta$-and $\gamma$-amino acids. Curr. Med. Chem. 2012, 19, 6178-6187. [PubMed]

18. Rodríguez-Mata, M.; García-Urdiales, E.; Gotor-Fernández, V.; Gotor, V. Stereoselective chemoenzymatic preparation of $\beta$-amino esters: Molecular modelling considerations in lipase-mediated processes and application to the synthesis of (S)-dapoxetine. Adv. Synth. Catal. 2010, 352, 395-406. [CrossRef]

19. Tasnádi, G.; Forró, E.; Fülöp, F. An efficient new enzymatic method for the preparation of $\beta$-aryl- $\beta$-amino acid enantiomers. Tetrahedron Asymmetry 2008, 19, 2072-2077. [CrossRef]

20. Tasnádi, G.; Forró, E.; Fülöp, F. Burkholderia cepacia lipase is an excellent enzyme for the enantioselective hydrolysis of $\beta$-heteroaryl- $\beta$-amino esters. Tetrahedron Asymmetry 2009, 20, 1771-1777. [CrossRef]

21. Tasnádi, G.; Forró, E.; Fülöp, F. Improved enzymatic syntheses of valuable $\beta$-arylalkyl- $\beta$-amino acid enantiomers. Org. Biomol. Chem. 2010, 8, 793-799. [CrossRef]

22. Forró, E.; Megyesi, R.; Paál, T.A.; Fülöp, F. Efficient dynamic kinetic resolution method for the synthesis of enantiopure 6-hydroxy- and 6-methoxy-1,2,3,4-tetrahydroisoquinoline-1-carboxylic acid. Tetrahedron Asymmetry 2016, 27, 1213-1216. [CrossRef]

23. Megyesi, R.; Mándi, A.; Kurtán, T.; Forró, E.; Fülöp, F. Dynamic kinetic resolution of ethyl 1,2,3,4-tetrahydro$\beta$-carboline-1-carboxylate: Use of different hydrolases for stereocomplementary processes. Eur. J. Org. Chem. 2017, 32, 4713-4718. [CrossRef]

24. Paál, T.A.; Forró, E.; Fülöp, F.; Liljeblad, A.; Kanerva, L.T. Lipase-catalyzed kinetic resolution of 1,2,3,4-tetrahydroisoquinoline-1-acetic acid esters. Tetrahedron Asymmetry 2008, 19, 2784-2788. [CrossRef]

25. Paál, T.A.; Forró, E.; Liljeblad, A.; Kanerva, L.T.; Fülöp, F. Lipase-Catalyzed kinetic and dynamic kinetic resolution of 1,2,3,4-tetrahydroisoquinoline-1-carboxylic acid. Tetrahedron Asymmetry 2007, 18, 1428-1433. [CrossRef]

26. Rangel, H.; Carrillo-Morales, M.; Galindo, J.M.; Castillo, E.; Obregón-Zúñiga, A.; Juaristi, E.; Escalante, J. Structural features of N-benzylated- $\beta$-amino acid methyl esters essential for enantiodifferentiation by lipase B from candida antarctica in hydrolytic reactions. Tetrahedron Asymmetry 2015, 26, 325-332. [CrossRef]

27. Pérez-Venegas, M.; Reyes-Rangel, G.; Neri, A.; Escalante, J.; Juaristi, E. Mechanochemical enzymatic resolution of N-benzylated- $\beta 3$-amino esters. Beilstein J. Org. Chem. 2017, 13, 1728-1734. [CrossRef]

28. Nagy, B.; Galla, Z.; Bencze, L.C.; Toșa, M.I.; Paizs, C.; Forró, E.; Fülöp, F. Covalently immobilized lipases are efficient stereoselective catalysts for the kinetic resolution of rac-(5-phenylfuran-2-yl)- $\beta$-alanine ethyl ester hydrochlorides. Eur. J. Org. Chem. 2017, 20, 2878-2882. [CrossRef]

29. Patel, R.N. Green Biocatalysis; Wiley: Hoboken, NJ, USA, 2016.

30. Zhang, X.-X.; Gao, Y.; Hu, X.-S.; Ji, C.-B.; Liu, Y.-L.; Yu, J.-S. Recent advances in catalytic enantioselective synthesis of fluorinated $\alpha$ - and $\beta$-amino acids. Adv. Synth. Catal. 2020, 362, 4763-4793. [CrossRef]

31. Zablocki, J.A.; Tjoeng, F.S.; Bovy, P.R.; Miyano, M.; Garland, R.B.; Williams, K.; Schretzman, L.; Zupec, M.E.; Rico, J.G.; Lindmark, R.J.; et al. A novel series of orally active antiplatelet agents. Bioorg. Med. Chem. 1995, 3, 539-551. [CrossRef]

32. Johnson, T.B.; Livak, J.E. Researches on pyrimidines. CXLIX. The synthesis of aryl substituted dihydrouracils and their conversion to uracil derivatives. J. Am. Chem. Soc. 1936, 58, 299-303. [CrossRef]

33. Forró, E.; Fülöp, F. New enzymatic two-step cascade reaction for the preparation of a key intermediate for the taxol side-chain. Eur. J. Org. Chem. 2010, 16, 3074-3079. [CrossRef]

34. Forró, E. New gas chromatographic method for the enantioseparation of $\beta$-amino acids by a rapid double derivatization technique. J. Chromatogr. A 2009, 1216, 1025-1029. [CrossRef]

35. Straathof, A.J.J.; Rekels, J.L.L.; Heijnen, J.J. Mass balancing in kinetic resolution: Calculating yield and enantiomeric excess using chiral balance. Biotechnol. Bioeng. 1995, 45, 536-538. [CrossRef] [PubMed]

36. Davies, S.G.; Fletcher, A.M.; Lv, L.; Roberts, P.M.; Thomson, J.E. Asymmetric synthesis of $\beta$-fluoroaryl- $\beta$-amino acids. Tetrahedron Asymmetry 2012, 23, 910-925. [CrossRef] 
37. Forró, E.; Paál, T.; Tasnádi, G.; Fülöp, F. A new route to enantiopure $\beta$-aryl-substituted $\beta$-amino acids and 4-aryl-substituted $\beta$-lactams through lipase-catalyzed enantioselective ring cleavage of $\beta$-lactams. Adv. Synth. Catal. 2006, 348, 917-923. [CrossRef]

38. Bull, S.D.; Davies, S.G.; Delgado-Ballester, S.; Kelly, P.M.; Kotchie, L.J.; Gianotti, M.; Laderas, M.; Smith, A.D. Asymmetric synthesis of $\beta$-haloaryl- $\beta$-amino acid derivatives. J. Chem. Soc. Perkin Trans 1 2001, 23, 3112-3121. [CrossRef]

Sample Availability: Samples of the compounds ( \pm )-2a-e, $( \pm)-\mathbf{3 a}-\mathbf{e},(\mathrm{R})-\mathbf{- 4 a}-\mathbf{e}$ and $(\mathrm{S})-\mathbf{5 a}-\mathbf{e}$ are not available from the authors.

Publisher's Note: MDPI stays neutral with regard to jurisdictional claims in published maps and institutional affiliations.

(C) 2020 by the authors. Licensee MDPI, Basel, Switzerland. This article is an open access article distributed under the terms and conditions of the Creative Commons Attribution (CC BY) license (http://creativecommons.org/licenses/by/4.0/). 